Aus dem pathologisch-anatomischen Institut Basel.

(Vorsteher: Prof. Dr. E. Hedinger.)

\title{
Über rezidivierende Encephalitis haemorrhagica, zngleich ein Beitrag zur Kenntnis der Encephalitis nach Appendicitis.
}

\author{
Von \\ Dr. Heinrich Heuber, \\ III. Assistenten.
}

Die Encephalitis haemorrhagica und die infektiös-toxische nicht eitrige Encephalitis überhaupt haben in den letzten. Jahren eine besonders intensive Bearbeitung erfahren. Meine Beobachtung von hämorrhagischer Encephalitis bietet namentlich nach zwei Seiten Interesse, erstens in ätiologischer und zweitens in rein morphologischer Beziehung.

Es handelt sich um einen 13 jähr. Knaben, dessen Vater an Gicht litt und dessen Mutter und Geschwister gesund sind. Der Knabe selbst sei in den ersten drei Lebensjahren ebenfalls immer gesund gewesen, sei auch, was seine körperlichen und geistigen Eigenschaften anbetrifft, ein völlig „,normales Kind" gewesen.

Mit 31/2 Jahren erkrankte er akut unter den Erscheinungen einer Appendicitis und wurde deshalb am vierten Tage der Krankheit in die chirurg. Klinik in Basel überführt. Aus der damaligen Krankengeschichte, die mir in liebenswürdiger Weise zur Verfügung gestellt wurde, ist zu entnehmen, daß das Kind beim Eintritt etwas schwächlich aussah und einen schwerkranken Eindruck machte. Die inneren Organe waren ohne Besonderheiten. Der Lokalstatus mit deutlicher Druckempfindlichkeit, entsprechender Resistenz und abgegrenzter Dämpfung, verbunden mit geringen allgemein-peritonitischen Erscheinungen, ließen eine Appendicitis acuta, event. mit beginnendem Abszeß diagnostizieren. Bei der sofort vorgenommenen Operation zeigt sich das Peritoneum der Bauchwand und der Dünndärme injiziert und mit Fibrin belegt. In der Bauchhöhle befindet sich etwas mit Fibrinflocken untermischte eitrige Flüssigkeit. Das Cöcum ist in entzündliche Schwarten eingelagert. Die Appendix liegt nach oben an der Vorderwand des Cöeums und ist stark geschwollen, injiziert und nahe der Basis gangränös.

Nach Appendektomie mit Spülung; Drainage und teilweisem Schluß der Wunde besserte sich der Zustand des Pat. wieder und die vorher $38,2^{\circ}$ 
betragende Temperatur sank auf $37,4^{0}$. Zwei Tage nach derOperation zeigte sich bei dem Knaben nach einem Besuch der Mutter ein ziemlich starker Aufregungszustand, dem nach ein paar Stunden plötzlich Zuckungen im linken Bein, im linken Arm und in der linken Schulter folgten. Der Kopf war dabei nach rechts gedreht, die Augen sahen nach rechts und nach oben. Die Sehnenreflexe der linken Seite waren gesteigert, der Babinskische Reflex links positiv. Das Kind sprach nicht und reagierte nicht auf Anrufen. Versuche, den Kopf nach links zu drehen, schienen schmerzhaft zu sein. Pupillen o. B., Temp. 37, $1^{0}$, Puls 98.

Am nächsten Tag war der Zustand morgens unverändert, der Pat. ließ unter sich gehen. Gegen Abend trat eine schlaffe Lähmung des linken Beines auf. Die Pulsfrequenz war sehr wechselnd, zeitwreise nur 60 .

Am folgenden Tage stellten sich Zuckungen im Gebiet des linken $N$. facialis ein. Das Kind reagierte auf Ansprechen, ohne jedoch Antwort zu geben und ließ nicht mehr unter sich gehen. Die Zunge wurde gerade heraus gestreckt. Der Kopf wurde immer noch nach rechts gedreht, die Augen wurden bewegt. Die Zuckungen im Arm verschwanden allmählich. Diese Erscheinungen führten zur Wahrscheinlichkeitsdiagnose einer autochthonen Thrombusbildung in einer Vene der rechten Rinde.

In den nächsten Tagen stellten sich schlaffe Lähmungen im linken Arm und im linken Facialisgebiet ein, während die Zunge immer unbeteiligt blieb. Die Temperatur schwankte zwischen $36,4^{\circ}$ und $37,6^{\circ}$. Nach und nach gingen diese Lähmungen in der gleichen Reibenfolge, wie sie gekommen waren, wieder zurück, und beim Austritt des Kindes aus dem Sp:tal, zwei Wochen nach der Operation, waren die nervösen Symptome fast vollständig verschwunden. Die Reflexe waren normal und die gelähmten Muskeln arbeiteten wieder. Einzig das Drehen des Kopfes nach links wurde noch als schmerzhaft angegeben.

Utber das spätere Befinden des Kindes orientiert eine weitere Krankengeschichte der chirurgischen Klinik in Basel. Das Kind schien nach seiner ersten Erkrankung wieder vollkommen hergestellt. Erst nach 1 1/2 Jahren wurde bemerkt, daß der Knabe hie und da Zuckungen in Armen und Beinen bekam. Diese Anfälle traten anfangs alle 2-3 Wochen einmal auf, wurden aber bald häufiger und intensiver, bis zu viermal im Tag. Die Anfälle waren zum Teil leicht, zum Teil schwer. Bei den leichten zappelte der Pat. gleichmäBig mit Armen und Beinen und hielt dabei den Kopf meistens nach rechts, seltener nach links gedreht. Das Bewußtsein blieb erhalten und Pat. gab auf Anrufen Antwort. Die Dauer der leichten Anfälle betrug 1-2 Minuten.

Die schweren Anfälle waren seltener. Wie die leichten fingen sie an mit gleichmäBigen Zuckungen in Armen und Beinen. Dann aber wurde das Kind bewußtlos und stieß Schaum aus dem Munde. Nach einem Augenblick vollkommener Ruhe begannen die Zuckungen von neuem, währenddem das Bewußtsein wieder langsam zurückkehrte und dann ganz allmählich die Krämpfe verschwanden. Während der Anfälle war der Kopf nach rechts gedreht, seltener nach links.; 
Ein Überwiegen der Zuckungen der einen Körperhälfte über die der anderen oder der einen Extremität gegenüber der anderen wurde nie deutlich beobachtet.

Als die schweren Anfälle häufiger und intensiver wurden, wurde das Kind im März 1913, d. h. im Alter von 7 Jahren, auf die chirurg. Klinik verbracht, wo nach einer gewissen Beobachtungszeit eine Trepanation vorgenommen wurde. Man eröffnete den Schädel über dem rechten $\mathrm{Pa-}$ rietale. Außer dem reichlich vorhandenen Liquor, der während der ganzen Operation abfloß, fanden sich weder an der Dura noch an der Pia noch an der Hirnsubstanz irgendwelche Veränderungen. Nach Exzision eines kleinen Stückchens aus der Rinde zur histologischen Untersuchung wurde der Schädel durch Zurückklappen der Dura und Wiederauflegen der herausgesägten Knochenlamelle verschlossen.

Am vierten Tage nach der Operation stellten sich die Anfälle wieder ein und zeigten bald den gleichen Umfang wie vorher. Im übrigen hatte das Kind den Eingriff gut überstanden, die Wunde war gut geheilt und die Temperatur stieg nur einmal über $37^{\circ}$.

Die mikroskopische Untersuchung der exzidierten Rindensubstanz ergab keine pathologischen Veränderungen irgendwelcher Art.

Der Erfolg der Trepanation war also nicht der gewünschte, wie es ja bei dem völlig negativen Lokalbefund bei der Operation zu erwarten gewesen war. Immerhin gaben die Angehörigen an, daß der Knabe in den Jahren 1914 und 1915 wenig Anfälle gehabt habe. Sie seien zwar hie und da krisenartig aufgetreten, nachher sei das Befinden oft bis zu zwei Monaten gut gewesen, ,so daß das Kind sogar ohne Schaden Karussell fahren konnte."

Im September 1917 trat auf einmal und ohne daß eine besondere Ursache angegeben werden konnte, eine starke Verschlimmerung ein. Die' Anfälle nahmen rapid zu, es stellten sich schließlich bis zu hundert solcher im Tage ein, und der Knabe, der bis dahin ziemlich gut und, ganz nett" gehen konnte, wurde vollkommen bettlägerig und immer unbeweglicher.

Im Februar 1918 zeigte sich, wiederum ohne erkennbaren Grund, eine erneute Vermehrung der Anfälle. In den folgenden Wochen war dann der Verlauf der Krankheit ein sehr wechselnder, indem Perioden mit vielen Anfällen und solche mit relativ wenigen, sich mehrmals gegenseitig ablösten. Aus dieser Zeit wird angegeben, daß die Anfälle deutlich die rechte Körperhälfte stärker in Mitleidenschaft gezogen haben. Die Dauer der Anfälle war immer sehr kurz ( $1 / 2-1-2$ Minuten).

Im April 1919 machte der Knabe eine Pneumonie durch. Er habe damals stark Fieber gehabt und die Krankheit habe zu einem Dekubitus geführt.

Im darauffolgenden Winter, am 4. XII. 1919, erkrankte der Pat. ganz akut unter den Erscheinungen einer Pneumonie der rechten Seite (Temperatur bis $39^{\circ}$ ). Bei der gerade bestehenden Grippeepidemie dachte man selbstverständlich an diese Ätiologie, obschon der Knabe schon seit langem das Bett nicht mehr verlassen hatte und auch niemand in der Familie grippekrank gewesen war. Während der ersten Tage dieser Pneu- 
monie wurden die Anfälle eher etwas stärker, nahmen aber nachher wieder ab und blieben vom 12. XII. an ganz aus. Am 17. XII. war der Knabe den ganzen Tag über in der Agone und starb abends 6 Uhr 10 Minuten.

Aus dem Protokoll der Sektion, die am folgenden Tage, 15 Stunden post mortem, vorgenommen worden ist, seien folgende Angaben mitgeteilt:

Mittelgroßes Kind in geringem Ernährungszustand. Der linke Arm und das linke Bein sind in Kontrakturstellung. Pupillen mittelweit, beidseits gleich. Subkutanes Fettgewebe spärlich. Magen und Därme sehr eng. Serosa glatt und glänzend. In der Cócum- und Appendixgegend ziemlich ausgedehnte Adhäsionen. Harnblase o. B. In der Bauchhöhle keine freie Flüssigkeit. Rippenknorpel o. B. Lungen wenig kollabiert und retrahiert. Pleura stellenweise leicht fibrinös belegt. Herz zeigt außer einer geringen Atrophie keine Veränderungen. Foramen ovale zu. Aorta aszendens und thoracica o. B.

Lungen weisen besonders im rechten Unterlappen eine Reihe teils subpleural gelegener, teils mitten im Gewebe zerstreuter, meist hämorrhagischer, $1 / 2-2-3-4 \mathrm{~cm}$ messender lobulärer, etwas körniger; brüchiger Herde auf. Das Lungengewebe dazwischen ist graurot, glatt und glänzend, völlig kompressibel. In den Lungenarterien sind in den Hauptstämmen wie in den kleineren Ästen dunkelgrauroté, das Lumen meist völlig verlegende, mit der Wand leicht verklebte brüchige Gerinnsel. Bronchiale und und untere cervikale Lymphdrüsen sind klein, wenig anthrakotisch.

Milz ist mittelgroß, braunrot, von normaler Konsistenz. Follikel klein, Trabekel deutlich. Nebennieren und Nieren o. B. Magen o. B.

Leber ist mittelgroß, mit glatter Oberfläche. Die Schnittfläche zeigt mäBig deutliche Zeichnung. Die Zentren der Acini sind meist insulär, die peripheren Partien sind stellenweise etwas trüb. Glissonsche Scheiden nicht verbreitet, Konsistenz normal. Gallenblase o. B.

Pankreas o. B., Harnblase o. B., Darm o. B.

Der Schädel ist symmetrisch, mittelgroß. Im Parietale rechts findet sich eine alte Trepanationsstelle. Der Trepanationsdefekt ist knöchern bedeckt, dér Schädelknochen ist ziemlich fest mit dem aufliegenden Knochen verwachsen. Dura ist von mittlerer Spannung, zart, gut bluthaltig. Auf der rechten Seite ist sie im Bereich der Konvexität des Frontalhirns fest mit der Pia mater verwachsen. Die weichen Häute sind an der Konvexität ziemlich stark getrübt. Im Subarachnoidealraum in mittlerer Menge klarer Liquor. Im Bereich des Gyrus frontalis superior und medius der rechten Seite sind die weichen Hirnhäute mit der Rinde fest verwachsen. Die Rinde ist hier auf Frontalschnitten stark verdünnt, im Mittel 1-2 mm dick, gelbbraun verfärbt. Die weiße Substanz ist in einer Ausdehnung von ca. $4-5 \mathrm{~cm}$ Breite und 2-3 cm Dicke partiell zystisch umgewandelt. Die Zysten sind unregelmäBig geformt und werden durch schmale, etwas derbe, gelb- 
braun verfärbte Septen voneinander getrennt. 'Die Seitenventrikel sind leicht erweitert, das Ependym etwas derb, die Plexus chorioidei gut bluthaitig. III. und IV. Ventrikel sind mittelbreit, ihr Ependym wie im Seitenventrikel. Auf Sagittalschnitten durch die linke Großhirnhemisphäre findet man im Bereich des Gyrus temporalis primus und secundus und zum Teil auch im Gyrus supramarginalis in einer Ausdehnung von $2-3 \mathrm{~cm}$ teils diffuse, teils punktförmige multiple Blutungen. Ähnliche Blutungsherde trifft man im Gyrus centralis anterior und posterior und im Gyrus orbitalis der linken Seite, allerdings in geringerer Ausdehnung. Im linken Thalamus opticus, ungefähr in der Höhe des Nucleus lateralis und medialis und in geringerem Grade im Pulvinar thalami rechts sind ebenfalls vielfach zusammenfließende pùnktförmige Blutungen, die eine Ausdehnang von ca. $1 \frac{1}{2} \mathrm{~cm}$ einnehmen.

Die bakteriologische Untersuchung ergab in Milzagar und Milzbouillon lange, grampositive Diplostreptokokken und ziemlich viel grampositive Stäbchen.

Die mikroskopische Untersuchung von Herz, Milz, Leber und Nieren ergab kein bemerkenswertes Resultat. In der Lunge finden sich die Alveolen in den zahlreichen kleinen pneumonischen Herden mit Leukocyten, roten Blutkörperchen und desquamierten Epithelien zum Teil vollständig angefüllt. Fibrin ist nur spärlich vorhanden: Die Alveolarsepten sind mäßig breit und stellenweise lymphocytär infiltriert. In den angrenzenden Alveolen ist vielfach Ödemflüssigkeit mit desquamierten Epithelien. Die Bronchien sind leer, ihr Epithel ist meist gut erhalten. An mehreren Stellen sind einzelne Gefäße vollständig thrombosiert. Die Thromben bestehen aus zusammengebackenen, meist schlecht erhaltenen roten Blutkörperchen, die im Zentrum vielfach $z u$ einer homogenen Masse umgewandelt sind und aus Blutplättchen und wenig Fibrin. Eine Organisation der Thromben ist nirgends eingetreten. Im Bereich der thrombosierten Gefäße sind die Alveolen dicht mit roten Blutkörperchen, vereinzelten Leukocyten und Ödemfluissigkeit angefüllt. Die roten Blutkörperchen sind meist miteinander verbacken. Die Pleura über diesen Stellen ist unverändert.

Das Gehirn wurde in toto in Formol fixiert. Zur histologischen Untersuchung wurden an verschiedenen Stellen Stücke der Gehirnsubstanz entnommen and in Celloidin eingebettet. Zur Färbung der Schnitte sind folgende Methoden angewandt worden: Hämalaun-Eosin, van Giesonsche Färbung, Weigertsche Elastinfärbung, Weigertsche 
Markscheidenfärbung, Färbung der Ganglienzellen nach Lenhossék, der Neuroglia nach v. Szüts, der Plasmazellen mit Methylgrün-Pyronin, nach Weigert modifizierte Gramfärbung. Zur Differenzierung von Leukocyten und Polyblasten diente die Oxydasereaktion an Gefrierschnitten.

Die bistologische Untersuchung des Gehirns hat folgenden Befund ergeben:

Die weichen Hirnhäute sind überall ziemlich stark hyperämisch und zeigen daneben in wechselndem Maße, besonders über den stärker beteiligten Hirnpartien, allerlei Veränderungen. Sie sind vielerorts ziemlich stark aufgelockert und hie und da von der Gehirnsubstanz abgehoben. Zwischen den Bälkchen des Subarachnoidealraumes finden sich stellenweise in diffuser Anordnung zahlreiche desquamierte Endothelzellen von ziemlicher Größe mit hellem Protoplasma und mäßig chromatinreichen runden mittelgroßen Kernen und ebenfalls ganz diffus verteilt in geringer Menge kleine runde Lymphozyten. An mehreren Orten, vor allem in einzelnen intergyralen Piasepten ist das Gewebe der weichen Hirnhäute herdweise dicht durchblutet und oft leukocytär infiltriert.

Die Gefäße der Pia sind stark erweitert. Ihr Inhalt besteht zum größten Teil aus roten Blutkörperchen. Dazwischen trifft man vielfach homogene und streifige Partien, die sich mit Eosin stark rot färben. Außerdem enthalten die Gefäße polynukleäre Leukocyten, die so zahlreich sein können, daß sie hier und da in dichten Haufen das ganze Lumen ausfüllen. Die Wandung der Gefäße ist intakt, in ihrer Umgebung ist keine besondere Infiltration. Die von der Gehirnoberfläche oder von den intergyralen Piasepten in die Gehirnsubstanz hineinziehenden kleinen Gefäße sind entweder unverändert, und zwar manchmal auch an Stellen, die direkt in stark vẹränderte Gebiete der Rinde hineinführen, oder man findet allerlei Veränderungen, die ganz denen der tiefer liegenden Gefäße entsprechen und weiter unten beschrieben werden sollen.

Die verschiedenen Partien des Gehirns zeigen im einzelnen folgende Veränderungen:

Auf Frontalschnitten durch den Herd im Gyrus frontalis sup. und medius der rechten Seite erkennt man im Gyrus front. sup. einen ca. $5: 1 \frac{1 / 2}{\mathrm{~cm}}$ großen, unregelmäßig buchtigen Hohlraum, in den vom Rand aus einzelne septenartige Stränge bineinragen. Im Gyrus front. med. finden sich mehrere kleinere, zum Teil miteinander zusammenhängende Lumina. Von der Oberfläche werden diese Räume noch durch eine $2-3 \mathrm{~mm}$ breite Rinde getrennt. Die Lupe zeigt, daß auch die kompakt erseheinende Gehirnsubstanz in der Umgebung der größeren Zysten von zahlreichen kleinen Lücken durchsetzt wird.

Mikroskopisch zeigen die Himhäute hier einen etwas derberen Bau als im übrigen Gehirn. Sie senden an der Verwachsungsstelle mit der Rinde mehrfach feine Bindegewebsfasern in deren oberste Schichten, die sich dort zu einem feinen Netz verflechten. Im übrigen sind sie gleichermaßen in- 
filtriert wie anderswo und sind auch etwas frisch durchblutet, während altes Blutpigment nirgends zu sehen ist.

Die Rindensubstanz zeigt ausgedehnte Sklerose mit dichtem Gliafaserfilz und zahlreichen kleinen und größerne Gliazellen, von denen die größeren oft eisenhaltiges Blutpigment enthalten. Dazwischen sind im Gewebe in unregelmäßiger Anordnung Herde mit aufgelockerter, kleinere und größere Lücken wabenartig einschließender Gliafaserung und daneben noch multiple etwas größere, ziemlich scharf begrenzte Hohlräume ohne Inhalt. In der Rinde wenig gut erhaltene Ganglienzellen, etwas mehr solche mit verwischten Konturen, unscharfem Kern und homogenem Protoplasma. Ferner sind, besonders in den gegen die Sulci zu liegenden Rindenpartien zahlreiche größere und kleinere pyramidenförmige Ganglienzellen, die mit Kalksalzen imprägniert sind und sich intensiv mit Hämalaun färoen. Ihre ebenfalls verkalkten Fortsätze lassen sich beim Gebrauch der Mikrometerschraube noch streckenweit im Gewebe verfolgen. Die Abgrenzung von Rinde und Mark ist unscharf. Das Mark ist wie die Rinde ausgedehnt sklerotisch verändert und weist dazwischen die gleichen stark lückenartig aufgelockerten Stellen auf. Seine Gliazellen enthalten stellenweise ziemlich reichlich eisenhaltiges Blutpigment. Bei Markscheidenfärbung lassen sich nur noch Reste markhaltiger Nervenfasern nachweisen.

Die Blutgefäße in Rinde und Mark sind gut gefüllt mit erhaltenén roten Blutkörperchen und stellenweise stark vermehrten Leukocyten. Die Gehirnsubstanz ist von der Gefäßwandung meist retrahiert und die dadurch entstehenden perivaskulären, sog. Rothschen Räume enthalten manchmal Erythrocyten und kleine runde Lymphocyten in geringer Menge und vereinzelte etwas größere Zellen mit ziemlich chromatinreichen unregelmäßig geformten Kernen, die wohl als gewucherte Elemente der Adventitia zu deuten sind. In diesem Raum, wie in der angrenzenden Gehirnsubstanz sind oft besonders deutliche Gliazellen mit Blutpigment enthaltendem Protoplasma. Der periadventitielle His'sche und der intraadventitielle Virchow-Robinsche Lymphraum sind kaum erweitert and selten etwas mit Blut gefüllt.

Im Gyrus temp. sup. links, wo makroskopisch die stärksten Veränderungen sind, sind die weichen Hirnhäute wie oben beschrieben veränd st, doch sind hier sowohl Blutung wie Infiltration mit Leukocyten besonders ausgesprochen. In der Gehirnrinde, vor allem in der Molekularschicht and in der Schicht der kleinen Pyramidenzellen befinden sich zahlreiche Blutungen im Gewebe, die zum Teil rund sind, zum Teil mehr länglich, radiär gegen das Mark zu verlaufen, zum Teil ziemlich unregelmäßig, aus mehreren konfluierten Herden bestehen. Thre Ausdehnung geht selten über 3-5 $\mathrm{mm}$ hinaus. An einzelnen Stellen reichen die Blutungen bis an die Gehirnoberfläche, wo sie öfters zusammenhängen mit Blutungen, die sich in den weichen Hirnhäuten oder darunter im epicerebralen Lymphraum ausbreiten. Die Blutungsherde liegen manchmal um ein kleines Gefäß herum, oder doch in der Nähe eines solchen, doch ist dieses Verhalten nicht konstant. Die einzelnen, dicht beisammen liegenden roten Blutkörperchen reichen 
dabei meistens dicht an die Gefäßwand heran. Nur ziemlich selten sieht man die typischen Ringblutungen oder Andeutungen von solchen, wo zwischen dem Gefäß und der Blutung eine von Blutzellen freie homogen aussehende, heller als die übrige Gehirnsubstanz gefärbte Zone sich befindet, in der man hier und da vereinzelte polyblastenähnliche Zellen trifft. Die Gefäße in solchen Ringblutungen sind Kapillaren, deren Inhalt eine mit Eosin stark rot gefärbte homogene hyaline Substanz bildet und deren Wandung oft etwas undeutlich erscheint. Sämtliche Blutungsherde enthalten außer den Erythrocyten weiße Blutzellen, fast ausschließlich Leukocyten, und zwar meistens nur in geringer Zahl, an vereinzelten Stellen jedoch in stark vermehrter Menge. In der Peripherie sind die Herde etwas unscharf begrenzt, indem die einzelnen Zellen lockerer beisammenliegen und sich allmählich in der Gehirnsubstanz verlieren.

Die Gefäße zeigen, was Inhalt und Wandung betrifft, wechselnde Verhältnisse. Die Kapillaren sind weit, in ihrem Lumen liegen oft bis drei rote Blutkörperchen nebeneinander, die meist gut voneinander abgegrenzt sind. Mancherorts enthalten die Kapillaren sehr reichlich, oft fast ausschlieBlich Leukocyten. Vielfach ist ihr Lumen auch angefüllt mit homogenen oder streifigen hyalinen Massen. Die Wandung der Kapillaren ist fast überall intakt.

Die etwas größeren Gefäße, d. h. die Arterien vom präkapillaren Typus und wohl in großer Mehrzahl die kleinen Venen sind selten ganz unverändert. Meist enthalten sie neben den roten Blutkörperchen in stark vermehrter Anzahl Leukocyten, die sich entweder nur in einer randständigen Zone finden oder ziemlich oft den größten Teil des Lumens einnehmen. In den Gefäßen, die die meisten Veränderungen aufweisen, findet man vielfach einen großen Teil der Lichtung mit den gleichen homogenen oder streifigen Massen wie in den Kapillaren erfüllt, um die herum im wechselndem Verhältnis gut erhaltene rote Blutkörperchen, Leukocyten und andère Zellen, die mit ihren länglichovalen chromatinarmen Kernen und ihrer länglichen, oft etwas gequollenen Form wohl als desquamierte oder gewucherte Endothelzellen zu betrachten sind. Die Wandung der Gefäße ist oft verbreitert, aufgesplittert. Die vaskulären Lymphräume, der intraadventitielle Virchow-Robinsche und der periadventitielle His'sche Raum, sind vielfach ziemlich weit und enthalten reichliche rote Blutkörperchen, Leukocyten und gewucherte Gefäßwandzellen mit plumpen, gelappten, chromatinrèichen Kernen und ganz spärlichem Protoplasma. Nach außen ist um die Gefäße und um die in die Lymphräume ausgetretenen Infiltratmassen das den His'schen Raum umschließende Endothel fast überall deutlich zu sehen, und nur vereinzelt ist diese scharfe Umgrenzung dadurch gestört, daß die roten Blutkörperchen in das umgebende Hirngewebe durchgebrochen sind. Die zog. Rothschen Räume, die durch Retraktion des Hirngewebes von der äußersten Gefäßschicht entstehen, sind in diesen Präparaten wenig ausgesprochen. Sie können den gleichen Inhalt aufweisen wie die vaskulären Lymphräume. Ziemlich spärlich sieht man Gefäße, in deren Lymphscheiden und in deren nächster Umgebung in der Gehirn- 
substanz man homogene, mit Eosin ganz hellrot gefärbte Massen findet, die mit großer Wahrscheinlichkeit als geronnenes eiweißhaltiges Exsudat anzusprechen sind und die in wechselnder Menge stärker tingierte feinfaserige fibrinähnliche Substanz und mancherorts reichlich Leukocyten einschließen.

An der Stelle der Blutungen ist keine Grundsubstanz mehr zu sehen, da die Zellen der Blutung eng beisammen liegen. Die Gehirnsubstanz ist im allgemeinen intakt, mit Ausnahme einiger unregelmäBig begrenzter Herde, in denen das Gewebe mehr oder weniger stark aufgelockert ist und eigentlich nur aus einem wabenartig angeordneten Gliafasernetz mit wenigen Kernen besteht. In der Umgebung einiger Gefäße und Blutungsherde ist die Gehirnsubstanz in mittlerem Grade diffus von polynukleären Zellen durchsetzt. Die Ganglienzellen scheinen an wenigen Stellen in der Nähe von stärker veränderten Gefäßen gequollen, ihre Ausläufer undeutlich und die Nißlschen Schollen an die Peripherie des Protoplasmas gedrängt oder ganz aufgelöst. Im übrigen sind die Ganglienzellen ohne besondere Veränderungen. Die Gliafasern und Gliazellen sind nicht merklich vermehrt. In dem an die Gefäße angrenzenden Gewebe findet man jedoch neben den kleinen Gliazellen oft etwas größere Zellen in mittlerer Menge mit runden blasigen Kernen und schmalem Protoplasma oder solche, die neben einem runden oder ovalen Kern von größerem Chromatingehalt ein deutliches Protoplasma aufweisen.

Die Gehirnsubstanz ist überall etwas retrahiert, so daß die sog. perizellulären und perivaskulären Räume deutlich hervortreten. In der Marksubstanz sind die Gefäße erweitert und enthalten vermehrte Leukocyten, sonst sind keine Veränderungen zu sehen.

Im Gyrus centralis anterior und posterior der linken Hemisphäre zeigen Hirnhäute wie Hirnsubstanz ähnliche Verhältnisse wie im Gyrus temporalis sup. dieser Seite. Die Hirnhäute sind sehr stark durchblutet und leukocytär infiltriert. Die Blutungen sind besonders im Gyurs centr. post. im unteren Drittel recht ausgedehnt, vielfach streifenförmig. Der Zusammenhang zwischen Blutungsherden und Gefäßen ist hier deutlicher, indem diese sehr oft im Innern der Blutungen, zentral oder mehr peripher, zu finden sind und oft den schon beschriebenen hyalin aussehenden Inhalt haben und hie und da von einem Hof von Leukocyten umgeben sind. Daneben trifft man mehrfach typische Ringblutungen in der Rinde und an einigen Stellen auch in dem sonst nicht weiter veränderten Mark. Die polynukleären Leukocyten, die in den Lymphräumen oft sehr reichlich vorkommen and manchmal schmale, keulenförmige, degenerierte (?) Kerne besitzen, sind auch in der Umgebung der Gefäße im Gewebe ziemlich zahlreich anzutreffen. Zwischen den einzelnen Blutungsherden sieht das Grundgewebe hie und da etwas aufgelockert aus, ist sonst aber gleich wie im Gyrus temporalis. Ganglienzellen ebenfalls wie im Temporallappen.

Ähnliche Verhältnisse wie im Gyrus temporalis sup. findet man auf Schnitten durch die ausgedehnten Blutungsherde im Thalamus der linken Seite. Vor allem fallen hier ziemlich ausgedehnte Herde mit Auf- 
lockerung der Gewebes auf. Etwas mehr ausgeprochen sind im Thalamus vielleicht auch solche Stellen, wie sie im Gyrus temporalis als mit Exsudat durchsetztes Gewebe beschrieben worden sind. Die Blutungsherde sind ziemlich reichlich; man erkennt in diesen Präparaten an quer- und längsgetroffenen Gefäßen besonders gut, wie die Blutungen nicht kleine, eng umschriebene Herde bilden, sondern die Gefäße oft auf Strecken hin mantelförmig umgeben. Auch hïer gibt es Stellen, wo die roten Blutkörperchen dicht an die Gefäßwand heranreichen und zum Teil mit den Blutungen in den Lymphräumen der Gefäße zusammenhängen, während sich an anderen Orten zwischen Gefäß und Blutung eine freie Zone befindet, so daß typische Ringblutungen zustande kommen. Im übrigen sind die Veränderungen in jeder Beziehung gleich wie in der beschriebenen Temporalwindung.

Im Gyrus rectus und im Pulvinar thalami trifft man hauptsächlich reine Hämorrhagien in die erweiterten vaskulären Lymphscheiden und ins Gewebe, während infiltrative, exsudative und degenerative Prozesse eigentlich selten sind.

Noch geringer sind die Befunde der übrigen untersuchten Schnitte, so im rechten Gyrus centralis antund post., im Ammonshorn, in der Gegend der vorderen und hinteren Vierhügel, an verschiedenen Stellen der Fossa rhomboidea und der inneren Kapsel. Außer starker Füllung der Gefäße, manchmal mit beträchtlicher Vermehrung der Leukocyten, findet man gewöhnlich nichts Abnormes außer hie und da erweiterten und zur Seltenheit mit Blut gefüllten vaskulären Lymphräumen. Ganz vereinzelt sind auch einige rote Blutkörperchen ins Gewebe ausgetreten, so an einer Stelle in der Gegend des Nucleus dorsalis des $N$. vagus.

Das Kleinhirn zeigt keine besonderen Veränderungen.

Die Plexus chorioidei weisen normales Epithel auf. Ihr Stroma ist stellenweise etwas aufgelockert und in geringem Grade diffus lymphocytär infiltriert. Die Gefäße sind stark gefüllt mit normalem Inhalt. gewiesen.

Bakterien wurden in den Schnitten nirgends mit Sicherheit nach-

Pathologisch-anatomische Diagnose: Grippe, alte und frische haemorrhagische Encephalitis, lobuläre Pneumonie, Thrombose der Lungenarterien. Stauung und Verfettung der Leber, Peritonitis chronica fibrosa circumscripta. Status post appendectomiam et trepanationem.

Die Sektion hat also zur Annahme einer alten und einer frischen Encephalitis geführt und wir haben nun vor allem zu untersuchen, $\mathrm{ob}$ und wie weit wir nach dem makro- und mikroskopischen Befund im Gehirn zu einer solchen Diagnose berechtigt sind. Bei der Beschreibung neuer Fälle ist dies schon deshalb wichtig, weil nach Vogt und Reichard gerade aus diesem Gebiet zahlreiche kasuistische Mitteilungen einer ungenauen Diagnosestellung wegen völlig unbrauchbar sind. Wir werden bei dieser Untersuchung allerdings verschiedenen 
Schwierigkeiten begegnen, die sich einmal von der noch nicht festgelegten Umschreibung des Begriffes einer Entzündung der Gehirnsubstanz herleiten. Aber auch wenn man sich, um dem zu entgehen, von vornherein einer der mannigfachen Definitionen der Encephalitis einfach anschließt und z. B. mit Homén verlangt, daß dazu neben degenerativen Prozessen auch exsudative und infiltrative, ev. auch proliferative Prozesse als integrierende Bestandteile des Gesamtvorganges beteiligt sein müssen, ist es immer noch die Beurteilung und Wertung der histologischen Bilder, die bei den verschiedenen Autoren großen Schwankungen unterworfen ist.

Bei der nicht eitrigen Encephalitis findet man ganz allgemein Hyperämie, Exsudatbildung, zellige Infiltration, reaktive Vorgänge der Umgebung, Zerfall des nervösen Gewebes und oft Neubildung von Kapillaren.

Morphologisch äußern sich diese Prozesse einmal an den Gefäßen. Diese sind stark gefüllt mit roten Blutkörperchen und in normalem Verhältnis oder in stark vermehrter Menge mit weißen Blutzellen, vorab Leukocyten. Die Gefäßwandung ist meist intakt, zeigt aber oft auch Auflockerung, Aufsplitterung und zellige Infiltration. Die vaskulären Lymphscheiden sind meist erweitert und enthalten Infiltratzellen. Häufig sind Wucherungsvorgänge an den Zellen der Adventitia und des Endothels. Seltener kommen Risse, Verdickungen, Verkalkungen, fettige Degeneration usw. der Gefäßwand vor. In manchen Venen, besonders in den kleineren und kleinsten, kann Thrombenbildung eintreten.

Die Exsudatbildung ist gewöhnlich nicht sehr ausgesprochen, doch ist sie hie und da nachzuweisen durch den Befund von amorphen fibrinösen Massen in den vaskulären und perivaskulären Lymphräumen oder auch frei im Gewebe. Nach Friedmann soll ein derartiges Exudat übrigens ein Merkmal der nicht eitrigen Encephalitis sein, während bei Hirnabszeß und Encephalomalacie ein dïnnes seröses, nicht gerinnendes Exsudat die Regel wäre.

Die zellige Infitration setzt - sich nach Vogt aus Elementen verschiedener Form und verschiedener Herkunft zusarmmen. Wie bei entzündlichen Vorgängen in anderen Organen findet man polynukleäre Leukocyten, Lymphocyten, Plasmazellen, Mastzellen und epitheloide Zellen, die nicht selten verfettet sind und sich aus umgewandelten Neurogliazellen herleiten.

Das nervöse Gewebe geht bei der Encephalitis die verschiedenen 
degenerativen Prozesse ein, wie sie in gleicher Form bei jeder Schädigung auftreten. Nach Flatau und Homén sollen sich diese Veränderungen erst nach einer gewissen Dauer der Entzündung einstellen.

Die weichen Hirnhäute beteiligen sich teilweise durch Ödem, Quellung, Infiltration und eventuelle Blutung an der Entzïndung der Gehirnsubstanz.

Eine häufige Begleiterscheinung der Encephalitis sind Blutungen in das Parenchym des Gehirns. Sie kommen vor als kleinste flohstichartige, meist multiple Hämorrhagien oder als große, vielfach kon fiuierte Herde. Früher hat man die Blutungen wohl als direkt entzündliche Vorgänge angesehen. Friedmann läßt sie noch gelten als Anfangs- und Einleitungsstadium der Encephalitis, während Spielmeyer bei der Wernickeschen Form die Blutungen als eine Wirkung von Gefäßveränderungen, alșo als Folge und nicht als Bestandteil der Entzündung erklärt. Später hat dann M. B. Schmidt als Unterscheidungsmerkmal zwischen entzündlichen Blutungen und den ihnen oft gleichenden Blutungen bei Sinusthrombose, Tumor, Meningealerkrankungen usw. die besondere Ringform der Blutungsherde bej Entzündung angegeben. Oeller dagegen, der auch bei nicht entzündlichen Blutungen die gleiche Ringform gesehen hat, ist der Ansicht, daß die Blatungen bei Entzündungen keine charakteristischen Merkmale gegenüber reinen Stauungsblutungen aufweisen. E. spricht ihnen überhaupt jede entzïndliche Genese ab, indem er sie nur als „Ausdruck einer verschieden zustande gekommenen Stauung ${ }^{6}$ ansieht.

Und nun die histologischen Verhältnisse in unserem Fall. Wir haben gesehen, daß vor allem in den stärker veränderten Partien des Gehirns neben den degenerativen Erscheinungen an den Ganglienzellen und eventuell auch an manchen Infiltratzellen deutliche exsudative und infiltrative Prozesse festzustellen sind, die, auch wenn sie nicht in allzu großer Intensität auftreten, unbedingt die Diagnose einer Encephalitis erlauben. Wir hätten somit die letzte, zum Tode führende Krankheit des Patienten als eine Encephalitis haemorrhagica, allerdings mit der Reserve, die man an den Ausdruck ,haemorrhagica“ stellen muß, der von Leichtenstern und $F$ ürbringer beschriebenen Grippenencephalitis anzuschließen, um so mehr als sie auch die gleiche Ätiologie aufweist. Die starke Beteiligung der Gehirnrinde und die neben der Entzündung reichlichen Blutungen sprechen ebenfalls für Grippeencephalitis, während die Prozesse in den Stammganglien dabei wohl etwas seltener vorkommen, aber absolut nicht dagegen sprechen. 
Was nun die erste, sich an die Appendicitis anschließende Krankheit betrifft, so fand sich als ihr Substrat bei der Sektion ein Herd im hinteren Drittel des rechten Gyrus frontalis sup. und medius. Durch dessen Lokalisation an der Stelle des Blickzentrums erklärt sich zwanglos die damalige konjugierte Deviation von Kopf und Augen nach rechts. Für die Motilitätsstörungen in Arm und Bein der linken Seite und im linken Facialisgebiet findet man die Erklärung in einer vorübergehenden Schädigung der benachbarten Zentralwindungen durch eine ursprïnglich größere Ausdehnung des Prozesses, durch Quellung, kollaterales Ödem, Zirkulationsstörungen u. a. m. Das tiefer liegende Hypoglossuszentrum war, wie die Krankengeschichte hervorhebt, nicht affiziert. Daß sich die Beteiligung der Zentralwindung zuerst durch Reizsymptome und erst nachher durch Lähmung äußerte, während das Blickzentrum schon von Anfang an gelähmt war, ist ein Vorkommnis, das sich bei verschiedenen Affektionen dieser Hirnregion häufig zeigt.

Die histologischen Veränderungen dieses Herdes lassen über das Wesen des ursächlichen Prozesses nichts Sicheres mehr entnehmen. Streng genommen kann man aus ihnen nur ganz allgemein auf eine mit Blutung kombinierte Schädigung der Gehirnsubstanz schließen, der dann eine reaktive Wucherung der Glia gefolgt ist. Zieht man noch die Entstehung der Gehirnaffektion während einer Infektion und die übrigen klinischen Angaben mit in Betracht, so bleiben von den verschiedenen Möglichkeiten der Entstehung eigentlich nur noch die Venenthrombose auf entzündlicher Basis und die Encephalitis. Diese beiden Prozesse, die nicht scharf voneinander zu trennen sind, sondern im Gegenteil zahlreiche Übergänge zeigen, haben meist die gleiche Anamnese und sehr häufig den gleichen klinischen Verlauf, so daß die Differentialdiagnose zwischen ihnen oft direkt unmöglich kein kann. In unserem speziellen Fall möchten wir aber, trotzdem seinerzeit klinisch eine Venenthrombose angenommen worden ist, entschieden für eine Encephalitis eintreten. Erstens war ein altes thrombosiertes Gefäß an entsprechender Stelle nicht mehr aufzufinden, und dann zeigten die Hirnhäute, die bei einer Thrombose meist ansgedehnt durchblutet werden, absolut keine entsprechenden Veränderungen mehr.

Wir hätten somit einen Fall einer Encephalitis im Anschluß an Appendicitis mit späterem Rezidiv nach Grippe vor uns.

Von Interesse scheint in unserem Fall vor allem die Ätiologie der Gehirnaffektion zu sein. Ganz allgemein kommen wie bei jeder Ent- 
zündung die Intoxikation und die Infektion in Betracht, während andere entzündungserregende Momente, besonders mechanische und thermische nicht ganz auszuschließen sind, den zwei erstgenannten gegenüber aber entschieden stark in den Hintergrund treten.

Als klassisches Beispiel für die toxisch entstandene Encephalitis gilt die durch chronischen Alkoholismus bedingte Polioencephalitis sup. acuta nach Wernicke. Es schließen sich ihr an Encephalitiden nach Vergiftung mit Fleisch, Würsten, Pilzen, nach Blei- und Schwefelsäurevergiftung, nach Vergiftung durch Nikotin, Leuchtgas, Kohlenoxyd, nach Salvarsan usw. Auch Fälle von Gehirnentzïndung infolge von Autointoxikation bei Darmerkrankungen und sogar bei Koprostase gehören hierher, und bei den mehrfach in der Literatur zu findenden Kombinationen von Tumor mit Encephalitis sind wohl ebenfalls, toxische Stoffe mit im Spiel. Wenn auch in letzter Zeit zahlreiche, nicht alle Bedingungen für eine Entzündung aufweisende Fälle aus-. geschieden werden mußten, so bleiben doch genügend sicher gestellte Fälle bestehen, um die Intoxikation als einen wichtigen ätiologischen Faktor der Encephalitis ansehen zu dürfen.

Was die Infektion betrifft, so läßt sich aus den sehr zahlreich beschriebenen Fällen entnehmen, daß im Grunde genommen bei jeder Infektionskrankheit eine Encephalitis entstehen kann. Allerdings finden sich bedeutende graduelle Unterschiede, indem z. B. eine Krankheit relativ häufig, eine andere dagegen fast nie derartige Gehirnkomplikationen nach sich zieht. Auch der Befund der betreffenden Krankheitserreger im Gehirn zeigt ein wechselndes Verhalten und steht vor allem auch in keinem festen Zusammenhang mit der Intensität der histologischen Veränderungen (E. Fränkel). Die Art und Weise wie die Infektionserreger das Gehirn beeinflussen, ist ebenfalls recht verschieden und kann durch direktes Angreifen des lebenden Virus auf das Hirngewebe, durch seine Wirkung auf die Gefäße, durch Blutveränderung, Stase usw. zustande kommen. Bei Bakterien, die nicht in den Körper eindringen, ist entweder eine Mischinfektion für die Encephalitis verantwortlich oder es sind die von den Bakterien gebildeten Toxine, die eine solche, Wirkung entfalten. Durch diese Annahme einer toxischen Wixkung der Krankheitserreger ist es selbstverständlich nicht mehr möglich, zwischen toxischen und infektiösen Encephalitiden scharf zu trennen. Man hat vielmehr fast immer Ubergänge oder noch besser Kombination der beiden ursächlichen Momente zu erwarten.

In der Ätiologie der Encephalitis spielen auch Traumen eine ge- 
wisse Rolle, wie das sowohl klinische wie experimentelle Erfahrung gezeigt hat. Thre Wirkung ist allerdings wohl nie ursächlich, sondern ausschließlich auslösend durch Schaffung eiñes Locus minoris resistentiae oder durch die Beeinflussung der Zirkulation zu denken. In ähnlichem Sinne kann vielleicht auch einmal einer psychischen oder nervösen Komponente geringe Wirkung zugestanden werden.

Und nun zur Ätiologie im speziellen Fall. Der ersten Encephalitis ging um 6 Tage eine Appendicitis voraus, die schon perforiert war und $\mathrm{zu}$ einer fibrinös-eitrigen Peritonitis geführt hatte. Andere schädigende Momente toxischer oder infektiöser Natur kamen in jenen Tagen nicht in. Betracht, mit Ausnahme vielleicht der Narkose. Wieviel Narkotikum der Patient damals erhalten hat, konnte nicht mehr in Erfahrung gebracht werden, doch ist es zum mindesten äußerst unwahrscheinlich, daß eine so kurz dauernde Chloroformäthernarkose noch nach Tagen ein derartiges Krankheitsbild veranlassen kann. Dagegen spricht auch der histologische Befund mit den Blutungsherden. Es bleibt also als Ursache der Encephalitis nur noch der appendicitischperitonitische Prozeß übrig. Da ja jede Infektion eine Entzündung der Gehirnsubstanz im Gefolge haben kann, ist das theoretisch gar nicht etwas Außergewöhnliches. Merkwürdig ist nur, daß bei der relativen Häufigkeit der Appendicitis derartige Komplikationen und Folgekrankheiten so selten vorkommen. In der umfangreichen Literatur iuber die Encephalitis wird nur ganz vereinzelt eine Appendicitis als Ätiologie angegeben.

Ein solcher Fall, der mit dem unsrigen eine gewisse Analogie aufweist und darum hier erwähnt werden soll, wurde in neuester Zeit von Homén veröffentlicht. Der Fall betraf ein 11/2jähriges Mädchen, das an Appendicitis und periappendicitischem AbszeB erkrankte, am vierten Tage der Krankheit appendektomiert wurde, am achten Tage Bewußtseinsverlust, klonische Zuckungen und tonische Spannungszustände besonders der linken Körperhälfte aufwies und am elften Tage starb. Bei der Sektion fand man im Gehirn zahlreiche entzündlich erweichte Partien mit multiplen punktförmigen Blutungen fast ausschließlich in der Rinde und ausgedehnte Venen- und Șinusthrombose. Mikroskopisch zeigten sich außer den Blutungsherden, unter denen auch solche von Ringform zu sehen waren, stellenweise geringgradige, hauptsächlich aus Leukocyten bestehende kleinzellige Infiltrate in den Lymphscheiden und nur selten im Gewebe. Reaktive Prozesse 
waren gering, Bakterien wurden nicht. gefunden. Homén denkt sich hauptsächlich in Rücksicht auf den negativen Bakterienbefund und die geringgradigen exsudativen und proliferativen Prozesse, verbunden mit ausgedehnten Degenerationserscheinungen an den Infiltratzellen die Krankheit, die er übrigens nicht restlos als reine Encephalitis anerkennt, vor allem auf toxischem Wege zustande gekommen. Die Toxine würden von den im Peritonealexsudat massenhaft vorhandenen anaeroben, meistens hochgradig toxigenen Bakterien herstammen oder noch eher eine kombinierte Wirkung von aeroben, besonders pyogenen und anaeroben Bakterien darstellen, die nach Homén besonders deletär sein soll.

In unserem Fall, bei welchem in der Appendicitis und Peritonitis sowohl für eine toxische wie für eine infektiöse Entstehung der Encephalitis genügend Grundlage vorhanden war, kann man über die Art der Gehirnschädigung nachträglich selbstverständlich keine Schlüsse mehr ziehen, da ja nicht das histologische Bild des frischen Prozesses, sondern nur noch dessen Ausgang, der Erweichungs- und Skleroseherd vorliegt. Immerhin scheint uns unser Fall doch bemerkenswert als neues Beispiel für eine Encephalitis mit appendicitisch-peritonitischer Ätiologie.

Fast 10 Jahre nach der ersten Gehirnaffektion erkrankte unser Patient im Laufe einer mit Pneumonie komplizierten Grippe von neuem an einer Encephalitis. Hier gibt die Ätiologie weniger Anlaß zu Bemerkungen, scheint doch gerade die Influenza oder ihre Erreger eine besondere Affinität zum Gehirn zu entfalten vor den meisten anderen Infektionskrankheiten. Weit weniger häufig dagegen ist das zweimalige Auftreten einer Encephalitis bei den gleichen Menschen. Rein zufällig ist ein solches Vorkommnis bei einer verhältnismäßig so seltenen Krankheit wohl nicht und man sucht deshalb nach einer passenden Erklärung dafür.

Eine Möglichkeit, und zwar die nächstliegende, wäre die, dảß durch den ersten Prozeß im Gehirn ein Locus minoris resistentiae geschaffen worden wäre im Sinne einer erhöhten Empfänglichkeit für spätere Infektionen. Diese Erklärung scheint für unsern Fall nicht ohne weiteres zu passen, man müßte denn wegen der verschiedenen Lokalisation des ersten und zweiten Prozesses den Begriff des Locus minoris resistentiae nicht nur auf den Locus laesionis anwenden, sondern dem ganzen Gehirn eine verminderte Resistenz zugestehen.

Eine andere Erklärungsmöglichkeit liegt in der Annahme einer 
von vornherein bestehenden erhöhten Disposition des Gehirns Infektionskrankheiten gegenüber. Eine derartige Annahme hätte insofern etwas für sich, als sie es einmal erklären würde, weshalb unser Patient auf verschiedene von außen stammende Infektionen zweimal mit einer Hirnentzündung reagiert hat. Sie würde es ferner begreiflich machen, weshalb das erstemal eine in dieser Beziehung wenigstens sonst harmlose Appendicitis derartige Folgen gehabt hat. Normalerweise ist das Gehirn zwar gegen infektiöse Einflüsse recht wenig empfindlich, sonst müßten bei der Masse der Infektionskrankheiten, bei denen nach Fränkel die betreffenden Erreger wohl in drei Vierteln der Fälle ins Gehirn gelangen, Encephalitiden unbedingt viel häufiger zu verzeichnen sein. Das schließt aber keineswegs aus, da $B$ bei einzelnen Menschen das Gehirn eine erhöhte Empfänglichkeit zeigen kann, gleicherweise wie auch andere Organe je nach dem Individuum verschiedene Disposition aufweisen. Beweisen können wir mit unserm Fall eine solche Annahme allerdings nicht. Es ist uns auch nicht möglich, aus der Literatur genügend dienliche Fälle anzuführen. Einzig eine Arbeit von Filatow, der über das Vorkommen von Encephalitis bei Bruder und Schwester berichtet, ließe sich eventuell in unserem Sinn verwerten und an eine, in diesem Fall familiäre, besondere Disposition des Gehirns denken. Unsere zweite Annahme scheint also bis jetzt sehr wenig gestüitzt. Trotzdem möchten wir sie aber nicht ohne weiteres aufgeben, sondern es späteren Erfahrungen überlassen, zu entscheiden, ob und wie weit sie zu Recht bestehen kann.

\section{Literaturverzeichnis.}

1. Eichhorst, Meningoencephalitis haemorrhagica. Virchows Arch. Bd. 151. - 1898.

2. Fila tow, Encéphalite contagieuse chez le frère et la soeur. Soc. de neurologie et de psych. 9. VIII. 1899. Revue neurol. 1900, zit. nach Oppenheim und Cassirer, Die Encephalitis.

3. Fränkel, E., Ưber das Verhalten des Gehirns bei akuten Infektionskrankheiten. Virchows Arch. 194. Suppl. 1908.

4. Friedmann, M., Encephalitis und HirnabszeB. Handbuch der path. Anatomie des Nervensystems von Flatau, Jacobsohn und Minor. Berlin 1904, Bd. 1 .

5. Fürbringer, Zur Kenntnis schwerer organischer Gehirnleiden im Gefolge von Influenza. Deutsche med. Wochenschr. 1892. 
6. Homén, Experimentelle und pathologische Beiträge zur Kenntnis der infektiös-toxischen, nicht eitrigen Encephalitis. Arb. aus d. path. Inst. d. Univeristät Helsingfors, N. F., Bd. 2, Heft 1 und 2.

7. Lang bein und Oeller, Klinisch-pathologische Beiträge zur Frage der akuten hämorrhagischen Encephalitis. Deutsche Zeitschr. f. Nervenheilk. 1910, Bd. 38 .

8. Leichtenstern, Die primäre akute hämorrhagische Encephalitis. Deutsche med. Wochenschrift 1892 .

9. Meyer, Oskar, Über akute genuine Encephalitis. Frankf. Zeitschr. f. Path., Bd. 5, Heft 3.

10. Nauwerk, Influenza und Encephalitis. Deutsche med. Wochenschr. 1895.

11. Oeller, Entstehung und Heilung von Hirnblutungen, ihre Stellung zur hämorrhagischen Encephalitis. Deutsehe Zeitschr. f. Nervenheilk. 1913, 47/48.

12. Oppenheim und Cassirer, Die Encephalitis. 2. Aufl. Wien 1907.

13. Oppenheim, Zur Prognose der Gehirnkrankheiten im Kindesalter. Berliner klin. Wochenschr. Nr. 12/13, 1901.

14. Rosenblath, Zur Pathologie der Encephalitis acuta. Deutsche Zeitschr. f. Nervenheilk. 1914.

15. Schilder, P., Bemerkungen über die Symptome eines Falles von Encephalitis cerebelli bei Scharlach: Deutsche Zeitschr. f. Nervenheilk. 1920, Bd. 66 .

16. Schmaus, Akute Myelitis. In Lubarsch. Erg. d. allg. Path. 1903/4.

17. Schmidt, M. B., Über Gehirnpurpura und hämorrhagische Encephalitis. Zieglers Beitr. Suppl: VII, Festschrift für Arnold.

18. Stadelmann, E., Ein Fall von Eucephalitis haemorrhagica nach Influenza verlaufend unter dem Bilde ein Apoplexia sanguinea. Deutsche Zeitschr. f. Nervenheilk. Nr. 18, 1900.

19. Stegmann, Über Encephalitis haemorrhagica acuta. Münchn. med. Wochenschr. 1902.

20. Strä ußler, Zur Ätiologie der akuten hämorrhagisehen Encephalitis. Wiener klin. Wochenschr. 1902.

21. Vogt, $\mathbf{H}$., Encephalitis non purulenta. In Lewandowskys Handbuch der. Neurologie, Bd. III. 